\title{
Mental Workload in Medicine: Foundations, Applications, Open Problems, Challenges and Future Perspectives
}

Luca Longo

Technological University Dublin, luca.longo@tudublin.ie

Follow this and additional works at: https://arrow.tudublin.ie/scschcomcon

Part of the Computer Sciences Commons

\section{Recommended Citation}

Longo, L. (2016). Mental Workload in Medicine: Foundations, Applications, Open Problems, Challenges and Future Perspectives. Computer-Based Medical Systems (CBMS) 29th. IEEE International Symposium, 30-24 June, 2016] doi:10.1109/CBMS.2016.36

This Conference Paper is brought to you for free and open access by the School of Computer Sciences at ARROW@TU Dublin. It has been accepted for inclusion in Conference papers by an authorized administrator of ARROW@TU Dublin. For more information, please contact arrow.admin@tudublin.ie, aisling.coyne@tudublin.ie,gerard.connolly@tudublin.ie.

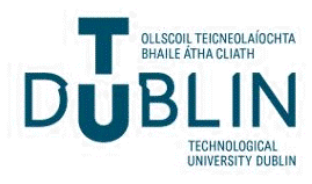




\title{
Mental workload in medicine: foundations, applications, open problems, challenges and future perspectives
}

\author{
Luca Longo \\ Dublin Institute of Technology: School of Computing, Dublin, Ireland \\ Email: luca.longo@dit.ie
}

\begin{abstract}
Mental workload is a design concept borrowed from Ergonomics with a significant adoption in the aviation and automobile industries. Nowadays, the consideration of this construct is also taking place in many modern clinical working environments for designing interacting and complex systems that impose ever greater cognitive demand on operators and less physical load. Measuring mental workload is essential for improving the interaction human-system, enhancing performance, reducing the operator's error and increasing safety. However, defining, measuring, assessing mental workload and understanding how this impinges on performance are still open problems. This secondary research is firstly aimed at introducing the construct of mental workload, its foundations, measurements techniques as well as applications in medicine. It then discusses open problems for applied research and eventually, it concludes with a list of challenges for scholars and practitioners. The goal is to provide the reader with a picture of the state of the science of mental workload in medicine and clinical domains with an eye towards future research.
\end{abstract}

Keywords-Mental workload; Medicine; Health-care; System design; Performance; Safety; Critical systems;

\section{INTRODUCTION}

Mental workload (MWL) is probably one of the most researched construct in Ergonomics [47, 57] and Human Factors [54] with several applications in aviation [27], automobile industry [4], rail industry [42] and increasingly considered in Human-Computer Interaction [36, 28] as well as clinical medicine $[24,32,5]$. This concept is invoked by scholars and practitioners when it is necessary to know the complexity of tasks that an operator has to perform, how busy an operator is and how s/he can handle additional tasks beyond those assigned. Additionally, the concept can be used to assess the operator's experience with the system under use and whether s/he is capable of dealing with unexpected events. The answers to these questions rely on the assumption that the MWL imposed by a given system on an operator can be measured. In a nutshell, MWL can be defined as the amount of cognitive work required for a person to complete a certain task over time. However, this is a simplistic view because mental workload "..emerges from the interaction between the requirements of a task, the circumstances under which it is performed and the skills, behaviours and perceptions of the operator" [20].
In the literature of MWL there are too many definitions and ad-hoc models subjectively adopted by practitioners, with an evident limitation both in their application across different domains and in their comparison [33, 34, 29].

This paper is organised as follows. Firstly, relevant notions related to mental workload are briefly introduced with a description of the most commonly adopted measurement techniques. A selection of applications of this construct in clinical medicine and health-care follows, aimed at showing how and why mental workload has been employed so far. A section is then focused on discussing open problems emerged by the review of published evidence followed by a proposal of research challenges and future perspectives.

\section{FOUNDATIONS AND MEASUREMENT TECHNIQUES}

Human Mental Workload is an important design concept and it is fundamental for exploring the interaction of people with technological devices [30]. It has a long history in Psychology with applications in Ergonomics and Human Factors, especially in the automotive [12] and aviation industries [20]. It has been largely reported that mental underload and overload can negatively influence performance [56]. On one hand, during information processing, when MWL is at a low level, individuals may frequently feel frustrated or annoyed. On the other hand, when MWL is at a high level, this can also be problematic and even dangerous as it can lead individuals to confusion, it can decrease their performance in processing information and increase the chances of mistakes. Hence, practitioners, interested in human or system performance, require answers about operator workload at all stages of system design and operation so design alternatives can be evaluated [20,7]. Unfortunately, although 50 years of research endeavour, MWL is still ill-defined without a universally accepted nor generally applicable definition. The measurement of MWL is an extensive area in which several assessment techniques have been proposed. Researchers in applied domains have demonstrated a tendency towards the use of ad-hoc or pool of mental workload measures justified by the multi-dimensional nature of the construct itself. Several reviews attempted to organise the significant 
amount of knowledge behind measurement procedures [56]. Generally, researchers agree in classifying MWL into three main broad categories [58, 47, 55, 7, 59]: self-assessment, task performance and physiological measures. The category of self-assessment measures is often referred to as subjective or self-report measures. It includes the direct subjective estimation of, for example, task difficulty or the state of the operator and it relies on the perceived experience of the interaction with an underlying interactive system. It is strongly believed that only the individual concerned with the task can provide an accurate judgement with respect to the MWL experienced, hence self-assessment measures have always attracted many practitioners. The class of task performance measures is based upon the assumption that the MWL of an operator, interacting with a system, gains relevance only if it influences system performance. These measures are based upon techniques aimed at directly quantifying the operator's capacity to perform the primary task at an acceptable level - low error likelihood and high efficiency - or indirect quantification of mental workload through quantification of the performance of a secondary task. The category of physiological measures takes into account physiological responses of the operator's body. These responses are believed to be correlated to MWL and are aimed at interpreting psychological processes by analysing their effect on the state of the body.

\section{APPLICATIONS IN MEDICAL DOMAINS}

The Mental Workload (MWL) construct has been applied to different medical specialities, both in simulation research and in practical studies, employing one or a combination of measurement techniques for various purposes. In the simulation conducted in [2], the NASA-TLX [20] - a well-known subjective measure - has been employed to evaluate the mental workload imposed by two different versions of a graphical cardiovascular display for improving detection of adverse cardiopulmonary events. NASA-TLX was also used for the investigation of how the substitution of electronic dry-erase whiteboards impacts the mental workload of clinicians of an emergency department [22] and for determining which factors influence the workload experienced by trauma team members during paediatric trauma resuscitations [41]. A modified version of the NASA-TLX was employed to investigate when to interrupt clinician workflow [48]. Similarly, an ad-hoc subjective measure was developed to quantify the mental workload experienced by residents for specific patient visits to an ambulatory care clinic [3]. The objective of the above two studies was to investigate how workload quantifications intervenes between clinical demand and the performance of health-care staff. Perception of mental workload was assessed with a seven-point Likert scale [53] to quantify the workload associated with different airway management procedures performed by different clinicians (faculty members, residents, community practitioners, nurses) and to suggest a more objective consideration of the non monetary costs of technical anaesthesia procedures. Subjective measures were employed by ward staff to test the workload imposed by electronic health records on clinicians during team conferences, ward rounds, nursing handovers and they were compared against paper-based records $[21,35,10,9]$.

Secondary task measures where employed during the performance of advanced laparoscopic tasks to measure the spare attentional resources of surgeons, with different level of training [61]. The objective was the quantification of their mental workload to confirm their level of expertise. Similarly, a secondary task measure - response time to a wireless vibrotactile device - was employed to measure the mental workload of anaesthetists during simulated practice [11] and clinical practice [6].

Primary tasks measures and subjective assessments of mental workload were jointly employed in different studies, for example, to test design alternatives of medical equipment applied to patient-controlled analgesia [26]. A time-in-motion analysis was performed in conjunction to the execution of the NASA-TLX questionnaire, in the context of an emergency department, to study the behaviour of faculty and resident physicians in the presence of an electronic whiteboard [16]. The NASA-TLX and a primary task performance measure were used in the context of intensive care to quantify the workload associated to the use of a prototype ecological display and two bar graph displays [15]. The goal was to analyse which display imposed less mental workload on nurses while identifying and treating oxygenation problems in an experimental laboratory simulation. Reaction time, response accuracy to underlying tasks were used as primary task measure, in conjunction to the NASA-TLX, to investigate which of four different interfaces of a novel anaesthetic monitoring system was imposing optimal mental workload to residents and fellows [8]. Similarly, primary task error rate detection and the NASA-TLX were used during simulator training on a complex laparoscopic task to identify those clinicians more likely to experience high workload and more prone to errors during skill transfer to the clinical environment [60].

Secondary task paradigms were employed to measure the mental workload imposed on students during a simulated consultation [39]. The goal was to study the human performance during complex dynamic tasks executed by anaesthesiologists in the operating theatres [17] and to investigate whether sleep deprivation increases cognitive workload during simulated surgical task [46]. The studies performed in [39] and [46] were conducted for educational purposes while [17] was executed in conjunction to subjective measures, for quantifying the spare capacity 
to perform another task and the vigilance of anaesthesia residents. The subjective Borg Workload Scale was employed with a secondary task measure - a response latency to a randomly activated alarm light - to study the effect of an electronic anaesthesia record keeping in the operating room and then compared to traditional manual recording procedures executed by clinicians [50]. Similarly, the Borg Scale was used with primary and secondary task measures to develop a technique of mental workload measurement of anaesthesiologists in operating rooms [51].

Physiological and subjective measurement techniques were also selected together for various purposes. For instance, in radiology, the NASA-TLX was used with physiological measures - gross eye and head movements to investigate the workload imposed by two display options - mammograms on a multi viewer adjacent to workstation and a digital version for soft copy display - on clinical readers to analyse mammograms [45]. A pilot study in an anaesthesia simulator environment, motivated by patient safety, was devoted to test the hypothesis that physiological measures such as heart rate, duration of fixation and pupil size increase, whereas saccade amplitude decreases with increased severity of a simulated critical incident, being induction of general anaesthesia the primary task executed by trainee anaesthetists [44].

Subjective, primary and secondary task measures as well as physiological measures were used together in few research studies to assess the cognitive load of clinicians during different phases of anaesthesia care and during teaching and non teaching cases [52]. This study was not only devoted to assess the mental workload of anaesthesia providers using multiple measures but also to investigate the impact of the task of teaching inexperienced clinicians on the mental workload of more senior residents or faculty anaesthesiologists during actual patient care, in the context of the traditional apprenticeship model. Another study, employing the three typologies of measurement - the NASA-TLX, physiological stress response and primary task performance - was devoted to the design of a framework for real-time monitoring of workload of physicians and for managing emergency department staff [25]. Yet, mental workload was assessed using the subjective Rating Scale of Mental Effort (RSME) in conjunction to primary task measures - time and movement efficiency - and a secondary task measure - reaction time on a vibrotactile stimulus-monitoring task during simulation-based surgical skills training [19].

\section{OPEN PROBLEMS}

The applications in medical settings clearly show the importance of the construct of Mental Workload for designing and developing interactive systems and clinical procedures aligned to the limited cognitive abilities of the
Table I

REVIEWED EVIDENCE BY MEASUREMENT TYPOLOGY AND DECADE

\begin{tabular}{|c|c|c|c|}
\hline Typology & 1990-1999 & 2000-2009 & $2010+$ \\
\hline self-assessment & $\begin{array}{l}{[17,40,51,} \\
50,26]\end{array}$ & $\begin{array}{l}53,52,16, \\
25,2,43,15, \\
21,45,8]\end{array}$ & $\begin{array}{l}{[60,35,41,} \\
49,48,9,10, \\
19,22]\end{array}$ \\
\hline $\begin{array}{l}\text { task performance } \\
\text { (primary/secondary) }\end{array}$ & $\begin{array}{l}{[17,51,50,} \\
26]\end{array}$ & $\begin{array}{l}{\left[\begin{array}{ll}52, & 16,25, \\
15,11]\end{array}\right.} \\
\end{array}$ & $\begin{array}{l}{[8,6,39,60,} \\
61,44,19]\end{array}$ \\
\hline physiological & [3] & {$[52,25,45]$} & [44] \\
\hline
\end{tabular}

human mind with the ultimate goal of predicting human and system performance. Table I summarises reviewed published evidence by measurement typology and decade, showing the increasing interest behind MWL assessment. Subjective self-assessment procedures have always appealed many practitioners because of their ease of implementation and use. However, a large amount of reviewed papers make use of the NASA Task Load Index [20], developed by the Human Performance Group at NASA's Ames Research Center. This subjective assessment instrument is freely available for non-commercial use and it has demonstrated high predictive validity across many research studies. However, it has been argued that its explosive use is just a Matthew effect ('The rich get richer' [37]) and its popularity is only explained because the tool has became the obvious choice [14].

The class of task performance measures, usually divided in primary and secondary task measures, assumes that the mental workload of an operator, when interacting with a system, acquires importance only if it influences system performance. In primary task methods, the performance of a clinician is monitored and analysed according to changes in the demands of the primary task under execution. Examples of common measurement parameters are response, reaction time, accuracy and error rate, estimation time, objective speed and signal detection [47]. In clinical settings, these measures represent a direct index of performance and they have been adopted because they are suitable for long single-tasks and periods of mental work. The main problem associated to them is their low ability in distinguishing performance of multiple parallels tasks. Secondary task measures are more useful for estimating the clinician's spare capacity to deal with additional tasks and are suitable for short tasks and period of mental work. However, the main drawback is their high degree of intrusiveness to the primary task, influencing the behaviour of the operator, and they are only sensitive to large changes in mental workload. Physiological measures represent natural mental workload indicators because, by definition, human work demands physiological activity. They are extremely good at monitoring this type of activity at a continuous interval, they have demonstrated high sensitivity and they do not interfere with the performance in the 
primary task. However, as table I suggests, only a small amount of studies has employed physiological measures and the reason is because they require tools and equipment that are often physically obtrusive. Moreover, analysis of data gathered by these tools is a non-trivial process, requiring well-trained experts. A minority of reviewed research studies have employed at least two measurement approaches $[51,50,26,16,15,8,45,60,46]$ while only three recent papers made use of all the three measurement techniques $[52,25,19]$. The reasons behind this are hidden in the complexity required by executing experiments, the difficulty in recruiting a significant enough amount of participants in clinical settings as well as the time and equipments required to perform such experiments.

Speculative interpretations of the construct of Mental Workload, ad-hoc applications and measurement techniques can be found in the literature. For instance, in a study in primary health-care centres, the notion of mental workload has been in somehow adopted to investigate the workload of physicians during diverse work activities [40] or considered as an indicator of performance for the evaluation of a prototype speech-input interface to an anaesthesia patient record in a simulated pilot study [1]. Other studies employed modified ad-hoc versions of the NASA-TLX respectively for the examination of the relationship of observed workflow interruptions with the perceived mental workload of hospital doctors during daily clinical shifts [49] and for the design of computerised clinical reminders [43]. An issue emerged from reviewed articles is the over-reliance on existing methods for mental workload assessment without a proper investigation of the validity of such methodologies. Several validation criteria have been proposed as guidelines [38, 31], suggesting that a methodology should:

- [sensitivity] have a high sensitivity to changes in resource demand or task difficulty and have good discriminatory capacity between significant MWL variations;

- [reliability] be reliable both within and across tests;

- [bandwidth] be capable of rapidly detecting transient changes in workload levels;

- [acceptability] have a high level of operator acceptance, showing at least face validity without being onerous;

- [diagnosticity] be highly diagnostic, capable of indicating the sources of variations in workload and quantifying their contributions by type/resource demand;

- [selectivity] be selectively sensitive to differences in resource demand and not to changes in factors unrelated to mental workload;

- [intrusiveness] not be intrusive or interfere with the performance of the task of the clinician, becoming a source of workload itself;

- [requirements] require the minimum possible equipment to avoid influencing the clinician's performance.

\section{Challenges And Future Directions}

In summary, mental workload is still a nebulous concept [57] defined in many ways with many associated dimensions. Reasons and advantages behind its measurement in medicine are clear, however there are many challenges for future research. Firstly, despite the explosive use of subjective measures in general, and the NASA-TLX in particular [14], self-report scales alone cannot capture mental workload [13]. Subjective perception of workload should be accompanied by performance measures on the primary task and possibly by physiological measures. In the upcoming years, thanks to advances in neuroscience and sensor technology, this challenge might be faced by employing novel pervasive and cheap equipment, increasingly available on the market, for gathering physiological bodily responses. Secondly, through these novel ways of gathering data, another challenge is the resolution of the 'envisioned world' problem and the consideration of the global operating environment as the collective source of information. Collecting relevant pieces of evidence related to mental worked should be performed in the centre of the clinician's natural operating environment and not only in experimental or simulating settings. Thirdly, a desirable mental workload assessment method should be developed and validated according to existing criteria [38, 31]. It should have high sensitivity, better if in a high bandwidth, low intrusiveness on the primary task and high reliability, as well as showing concurrent and convergent validity. Additionally, a measure should be used across many research and test settings, leading to the development of standards, and probably, to a better understanding and definition of the measure itself. Eventually, filtering and aggregating the relevant pieces of evidence toward a meaningful index of mental workload is the ultimate challenge for ergonomists and researchers. The goal should be the adoption of knowledge representation and reasoning techniques, borrowed from Artificial Intelligence [18], as well as machine learning and data analytical methodologies emerged in the last decade. The former might be appropriate for representing and modelling mental workload while the latter for automatically learning from data and perform accurate predictions of human performance [23].

\section{REFERENCES}

[1] A. Alapetite. Speech recognition for the anaesthesia record during crisis scenarios. Int. J. Med. Inform., 77(7):448-460, 2008.

[2] R. Albert, J. Agutter, N. Syroid, K. Johnson, R. Loeb, and D. Westenskow. A simulation-based evaluation of a graphic cardiovascular display. Anesth. Analg., 105(5):1303-1311, 2007.

[3] D. Bertram, D. Opila, J. Brown, S. Gallagher, R. Schifeling, I. Snow, and C. Hershey. Measuring physician mental workload: reliability and validity assessment of a brief instrument. Medical Care, 30(2):95-104, 1992. 
[4] K. A. Brookhuis and D. de Waard. Assessment of drivers' workload: Performance, subjective and physiological indices. In P. A. Hancock and P. A. Desmond, editors, Stress, Workload and Fatigue, pages 321-333. Mahwah, NJ: Lawrence Erlbaum, 2000.

[5] A. Byrne. Measurement of mental workload in clinical medicine: A review study. Anesth. Pain Med., 1(2):9094, 2011.

[6] A. Byrne, M. Oliver, O. Bodger, W. Barnett, D. Williams, H. Jones, and A. Murphy. Novel method of measuring the mental workload of anaesthetists during clinical practice. Br. J. Anaesth., 105(6):767771, 2010.

[7] B. Cain. A review of the mental workload literature. Technical report, Defence Research \& Dev. Canada, Human System Integration, 2007.

[8] S. Charabati, D. Bracco, P. Mathieu, and T. Hemmerling. Comparison of four different display designs of a novel anaesthetic monitoring system, the 'integrated monitor of anaesthesia (ima)'. Br. J. Anaesth., 103(5):670-677, 2009.

[9] L. Colligan, H. Potts, C. Finn, and R. Sinkin. Cognitive workload changes for nurses transitioning from a legacy system with paper documentation to a commercial electronic health record. Int. J. Med. Inform., 84(7):469-476, 2015.

[10] G. Dal Sasso and D. Barra. Cognitive workload of computerized nursing process in intensive care units. Comput. Inform. Nurs., 33(8):339-345, 2015.

[11] D. H. J. Davis, M. Oliver, and A. J. Byrne. A novel method of measuring the mental workload of anaesthetists during simulated practice. Br. J. Anaesth., 103(5):665-669, 2009.

[12] D. de Waard. The measurement of drivers' mental workload. The Traffic Research Centre VSC, University of Groningen, 1996.

[13] D. de Waard and B. Lewis-Evans. Self-report scales alone cannot capture mental workload. Cognition Technology and Work, 16(3):303-305, 2014.

[14] J. de Winter. Controversy in human factors constructs and the explosive use of the nasa-tlx: a measurement perspective. Cogn. Tech. Work, 16(3):289-297, 2014.

[15] J. Effken, R. Loeb, Y. Kang, and Z. Lin. Clinical information displays to improve icu outcomes. Int. J. Med. Inform., 77(11):765-777, 2008.

[16] D. France, S. Levin, R. Hemphill, K. Chen, D. Rickard, R. Makowski, I. Jones, and D. Aronsky. Emergency physicians' behaviors and workload in the presence of an electronic whiteboard. Int. J. Med Inform, 74(10):827-837, 2005.

[17] D. Gaba and T. Lee. Measuring the workload of the anesthesiologist. Anesth. Analg., 71(4):354-361, 1990.

[18] Y. Gil, M. Greaves, J. Hendler, and H. Hirsh. Amplify scientific discovery with artificial intelligence. Science,
346(6206):171-172, 2014.

[19] F. Haji, D. Rojas, R. Child, S. de Ribaupierre, and A. Dubrowski. Measuring cognitive load: performance, mental effort and simulation task complexity. Medical Education, 49(8):815-827, 2015.

[20] S. G. Hart. Nasa-task load index (nasa-tlx); 20 years later. In Human Factors and Ergonomics Society Annual Meeting, volume 50. Sage Journals, 2006.

[21] M. Hertzum and J. Simonsen. Positive effects of electronic patient records on three clinical activities. Int. J. Med. Inform., 77(12):809-817, 2008.

[22] M. Hertzum and J. Simonsen. Effects of electronic emergency-department whiteboards on clinicians' time distribution and mental workload. Health Informatics J., 22(1):3-20, 2016.

[23] M. I. Jordan and T. M. Mitchell. Machine learning: Trends, perspectives, and prospects. Science, 349(6245):255-260, 2015.

[24] J. Leedal and A. Smith. Methodological approaches to anaesthetists' workload in the operating theatre. $B r . J$. Anaesth., 94(6):702-709, 2005.

[25] S. Levin, D. France, R. Hemphill, I. Jones, K. Chen, D. Rickard, R. Makowski, and D. Aronsky. Tracking workload in the emergency department. Human Factors, 48(3):526-539, 2006.

[26] L. Lin, R. Isla, K. Doniz, H. Harkness, K. J. Vicente, and D. J. Doyle. Applying human factors to the design of medical equipment: Patient-controlled analgesia. $J$. Clin. Monit. Comput., 14(4):253-263, 1998.

[27] S. Loft, P. Sanderson, A. Neal, and M. Mooij. Modeling and predicting mental workload in en route air traffic control: Critical review and broader implications. Human Factors, 49(3):376-399, 2007.

[28] L. Longo. Human-computer interaction and human mental workload: Assessing cognitive engagement in the world wide web. In INTERACT (4), pages 402405, 2011.

[29] L. Longo. Formalising human mental workload as nonmonotonic concept for adaptive and personalised webdesign. In UMAP, pages 369-373, 2012.

[30] L. Longo. Formalising Human Mental Workload as a Defeasible Computational Concept. PhD thesis, Trinity College Dublin, 2014.

[31] L. Longo. A defeasible reasoning framework for human mental workload representation and assessment. Behaviour \& Information Technology, 34(8):758-786, 2015.

[32] L. Longo. Designing medical interactive systems via assessment of human mental workload. In Int. Symposium on Computer-Based Medical Systems, pages 364365, 2015.

[33] L. Longo and S. Barrett. Cognitive effort for multiagent systems. In Int Conf. on Brain Informatics, BI, pages 55-66, 2010. 
[34] L. Longo and S. Barrett. A computational analysis of cognitive effort. In Intelligent Information and Database Systems, Part II, pages 65-74, 2010.

[35] L. Longo and B. Kane. A novel methodology for evaluating user interfaces in health care. In Int. Symposium on Computer-Based Medical Systems, pages 1-6, 2011.

[36] L. Longo, F. Rusconi, L. Noce, and S. Barrett. The importance of human mental workload in web design. In Int. conf. on Web Information Systems and Technologies, pages 403-409, 2012.

[37] R. Merton. The matthew effect in science. the reward and communication systems of science are considered. Science, 159(3810):56-63, 1968.

[38] R. D. O' Donnel and T. F. Eggemeier. Workload assessment methodology. In K. Boff, L. Kaufman, and J. Thomas, editors, Handbook of perception and human performance, volume 2, pages 42/1-42/49. New York, Wiley-Interscience, 1986.

[39] M. Oliver, D. Davis, P. Jones, V. Rowe, and A. Byrne. Use of a secondary task paradigm to measure medical student's mental workload during a simulated consultation. Int. J. Clin. Skills, 4(2):92-95, 2010.

[40] P. Orozco and E. Garcia. The influence of workload on the mental state of the primary health care physician. Fam. Pract., 10(3):277-82, 1993.

[41] S. Parsons, E. Carter, L. Waterhouse, A. Sarcevic, K. O'Connell, and R. Burd. Assessment of workload during pediatric trauma resuscitation. J. Trauma Acute Care Surg., 73(5):1267-1272, 2012.

[42] L. Pickup, J. R. Wilson, S. Sharples, B. Norris, T. Clarke, and M. S. Young. Fundamental examination of mental workload in the rail industry. Theoretical Issues in Ergonomics Science, 6(6):463-482, 2005.

[43] J. Saleem, E. Patterson, L. Militello, S. Anders, M. Falciglia, J. Wissman, E. Roth, and S. Asch. Impact of clinical reminder redesign on learnability, efficiency, usability, and workload for ambulatory clinic nurses. J. Am. Med. Inform. Assoc., 15(5):632-640, 2007.

[44] C. M. Schulz, E. Schneider, L. Fritz, J. Vockeroth, A. Hapfelmeier, M. Wasmaier, E. F. Kochs, and G. Schneider. Eye tracking for assessment of workload: a pilot study in an anaesthesia simulator environment. Br. J. Anaesth., 106(1):44-50, 2011.

[45] S. Taylor-Phillips, M. Wallis, and A. Gale. Should previous mammograms be digitised in the transition to digital mammography? Eur. Radiol., 19(8):1890-1896, 2009.

[46] J. Tomasko, E. Pauli, A. Kunselman, and R. Haluck. Sleep deprivation increases cognitive workload during simulated surgical tasks. Am. J. Surg., 203(1):37-43, 2012.

[47] P. S. Tsang. Mental workload. In W. Karwowski, editor, Encyclopedia of Ergonomics and Human Factors, volume 1, chapter 166. Taylor \& Francis, 2006.
[48] M. Weigl, A. Müller, P. Angerer, and F. Hoffmann. Workflow interruptions and mental workload in hospital pediatricians: an observational study. BMC Health Serv. Res., 14(433):1-7, 2014.

[49] M. Weigl, A. Müller, C. Vincent, P. Angerer, and N. Sevdalis. The association of workflow interruptions and hospital doctors' workload: a prospective observational study. BMJ Qual. Saf., 21(5):399-407, 2012.

[50] M. Weinger, O. Herndon, and D. Gaba. The effect of electronic record keeping and transesophageal echocardiography on task distribution, workload, and vigilance during cardiac anesthesia. Anesthesiology, 87(1):144155, 1997.

[51] M. Weinger, O. Herndon, M. Zornow, M. Paulus, D. Gaba, and L. Dallen. An objective methodology for task analysis and workload assessment in anesthesia providers. Anesthesiology, 80(1):77-92, 1994.

[52] M. Weinger, S. Reddy, and J. Slagle. Multiple measures of anesthesia workload during teaching and nonteaching cases. Anesth. Analg., 98(5):1419-1425, 2004.

[53] M. Weinger, A. Vredenburgh, C. Schumann, A. Macario, K. Williams, M. Kalsher, B. Smith, P. Truong, and A. Kim. Quantitative description of the workload associated with airway management procedures. Clin. Anesth., 12(4):273-282, 2000.

[54] C. Wickens. Multiple resources and mental workload. Human Factors, 50(3):449-455, 2008.

[55] G. F. Wilson and T. F. Eggemeier. Mental workload measurement. In W. Karwowski, editor, Int. Encyclopedia of Ergonomics and Human Factors (2nd ed.), volume 1, chapter 167. Taylor \& Francis, 2006.

[56] B. Xie and G. Salvendy. Review and reappraisal of modelling and predicting mental workload in single and multi-task environments. Work and Stress, 14(1):74-99, 2000.

[57] M. S. Young, K. A. Brookhuis, C. D. Wickens, and P. A. Hancock. State of science: mental workload in ergonomics. Ergonomics, 2015, 1(58):1-17, 2015.

[58] M. S. Young and N. A. Stanton. Mental workload. In N. A. Stanton, A. Hedge, K. Brookhuis, E. Salas, and H. W. Hendrick, editors, Handbook of Human Factors and Ergonomics Methods, chapter 39, pages 1-9. 2004.

[59] M. S. Young and N. A. Stanton. Mental workload: theory, measurement, and application. In W. Karwowski, editor, Encyclopedia of ergonomics and human factors, volume 1, pages 818-821. Taylor \& Francis, 2006.

[60] Y. Yurko, M. Scerbo, A. Prabhu, C. Acker, and D. Stefanidis. Higher mental workload is associated with poorer laparoscopic performance as measured by the nasa-tlx tool. Simul Healthc., 5(5):256-271, 2010.

[61] B. Zheng, M. Cassera, D. Martinec, G. Spaun, and L. Swanström. Measuring mental workload during the performance of advanced laparoscopic tasks. Surg Endosc., 24(1):45-50, 2010. 